\title{
Optimization of automotive cooling systems characteristics using finite element analysis
}

\author{
George Gabriel Chiriac ${ }^{1 *}$, and Catalin Gabriel Dumitras ${ }^{1}$ \\ "'Gheorghe Asachi" Technical University of Iasi, Romania
}

\begin{abstract}
Due to a continuous evolving automotive industry, car manufacturers continuously develop new technologies to stay competitive on the market. Most of the modern cars are equipped with electronic devices used for functional or multimedia purposes. Electric cars have additional components used for charging and battery management. All the electronic components used in cars dissipate heat during functioning and all of them need cooling in order to keep a proper functioning temperature. Cold plates are a very efficient cooling solution used in most of the cars. The advantage of the cold plates is that it can dissipate a high amount of heat, have a small volume and can be placed in closed or tight areas. In order to enhance the thermal transfer between the cold plate and the cooling fluid, turbulence enhancement geometries are used inside the cold plate. The purpose of this paper is to use finite element analysis to compare different positions of one protrusion in the cooling channel to enhance the cooling process. The goal is to see if the protrusion position influences the cooling and which placement is more efficient. The second evaluation criteria of the efficiency is to keep a low-pressure drop inside the system.
\end{abstract}

\section{Introduction}

The automotive industry in a continuous expansion and rapid innovation of the technologies used inside the cars. The goal is to offer modern and innovative products, to continuous upgrade the existing technologies or develop new products in order the stay competitive on the market.

In the last years, we saw an electrification of the cars. Starting with the electronic devices that are placed inside the cars to increase the comfort of the driver or occupants and continuing with the electronics devices that are used to increase the safety of the car or to increase the ease of use, the amount of electronic components or electronic devices used to build cars increased significantly. Beside these features mentioned above, electrical cars have additional electrical components, because they are equipped with additional batteries, charging and battery management systems.

During the functioning of all the electronic components, Joule heating phenomenon appears and all the products are heated. For most of the components, the temperature can reach values that are not proper for functioning. Because of this reason, cooling devices needs to be used.

${ }^{*}$ Corresponding author: chiriacgeorgegabriel@yahoo.com 
Liquid cooling is one of the most efficient cooling methods used inside cars. In order to realize the cooling, pipes or cold plates filled with cooling liquid are used to absorb and transfer the heat away from the heat source [1]. When designing a cold plate, many requirements needs to be fulfilled. Starting with the total power dissipated by the electrical products, allocated space, pressure drop limit, mechanical requirements, but also manufacturing technologies and price, these requirements shows us that the cold plates need to be optimized even more in order the fulfill them.

The main purpose of the cold plate is to transfer the heat from the heat source into the cooling fluid, which will transport the heat away from the system [2]. The goal of this paper is to compare different position for one protrusion inside the cold plate and see the effect on the heat source temperature. Finite element analysis was used to compare the positions, evaluate the temperature of the heat source and the pressure drop of the system, in order to see the effect of each geometry.

\section{Synthetic analysis of the existing solutions}

J. Lee and K. S. Lee [3] studied the effect of protrusions and dimples on the heat transfer efficiency. Their conclusion was that the repetitive geometry of dimples and protrusions enhance the overall heat transfer from solid to fluid, but also it induces a large pressure drop between the starting and end of the heat exchanger. In their conclusions, they suggested that, in order to get the best heat transfer and pressure drop characteristics, the placement of the dimples and protrusions should be optimized.

In Fig. 1 can be observed that after each protrusion, a vortex is generated. When a vortex is generated, the fluid boundary layer is interrupted and the fluid stands more time in that volume. Because of the increase time, the fluid temperature increases, reducing the cooling effect of the solid nearby [4].

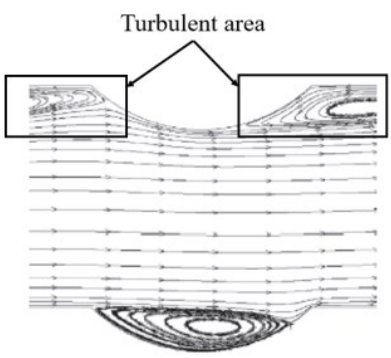

Fig. 1. Turbulent flow before and after a protrusion [3]

M. Faizan et al [5] confirm in their study that semi-spherical protrusions have a cooling enhancement effect on fluid cooled channels. They concluded that the benefit of improved cooling is caused by the increased mixing level of the fluid. In the same time, one of their concern is the pressure drop, since an array of dimples will significantly increase the pressure drop of the system and more pumping power is required.

Y. Xie et al [6] studied multiple shapes of dimple and protrusions placed inside a fluid cooled cold plate. In Fig 2 the temperature distribution over a hemispherical protrusion can be observed for different Reynolds numbers. In all four scenarios, even for low Reynolds number, we can see that the front surface of the protrusion, considering the fluid flow directions, is better cooled than the backside of the protrusion. Based on the temperature distribution that Y. Xie et al. presented, for low Reynolds numbers, the back surface of a protrusion and the surface right after are not so efficiently cooled as the front area. Therefore, if the heat up of the power source is not uniform and hot spots may appear, the position of the dimples is very important in optimizing the cooling effect. 


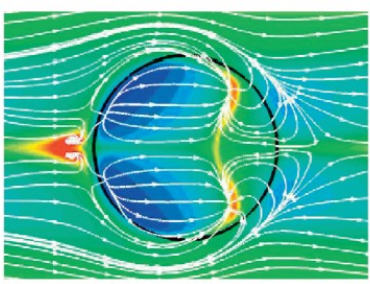

(a) $R e=3000$

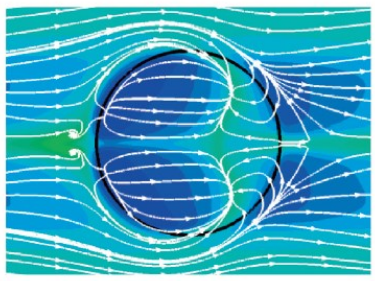

(c) $R e=7000$

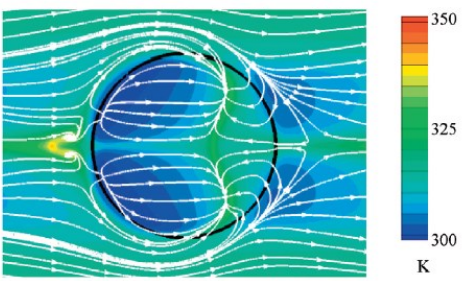

(b) $R e=5000$

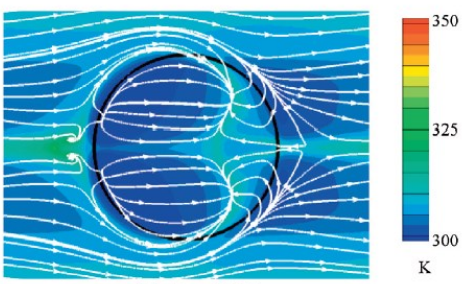

(d) $\operatorname{Re}=9000$

Fig. 2. Streamlines and temperature plot on the surface of hemispherical protrusions [6]

For high Reynolds numbers the cooling effect is similar for the entire area of the protrusions and the position of the protrusion in relation with the hot spot is less important.

J. Liu et al [7] also studied the effect of the protrusions on heat transfer enhancement. In Fig. 3 can be seen the temperature plot of their numerical simulations. Case A represents the test scenario with only a dimple. Test case B2 has an additional secondary protrusion with a height of $2 \mathrm{~mm}$ and a diameter of $20 \mathrm{~mm}$. For the test case B2 and B3, the height of the secondary protrusion, that can be found in the left side of the primary dimple increase to 4 $\mathrm{mm}$ and $6 \mathrm{~mm}$.

The temperature plot shows higher temperatures after each protrusion, the same behavior mentioned before. They saw on overall improvement in cooling due to addition of the protrusions. The height of the secondary protrusions are influencing the cooling behavior in the dimple area.

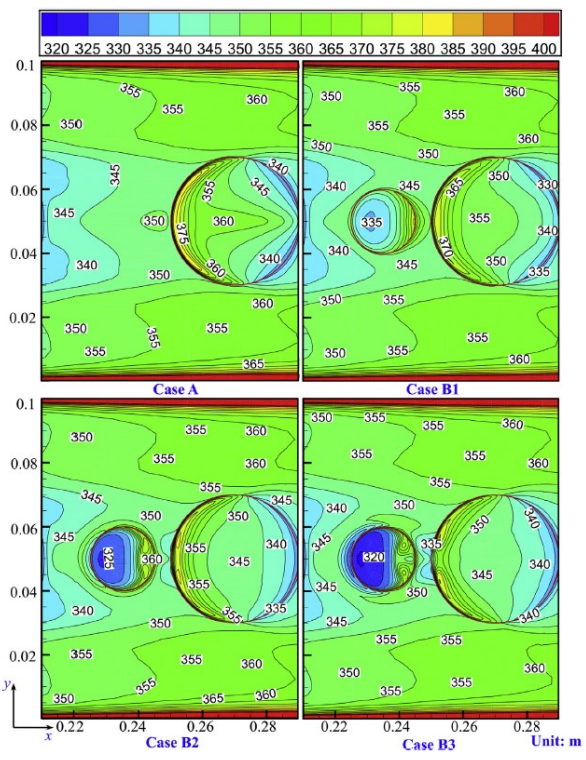

Fig. 3. Temperature contours on dimples and protrusions [7] 
The overall cooling is improved because they applied in their numerical simulations a contract heat flux of $1000 \mathrm{~W} / \mathrm{m}^{2}$ on the channel section. Therefore, a uniform applied power will not generate any hot spot that may be affected by the position of the protrusion. The protrusion will improve the cooling effect on any position, if it's placed on a surface without any hot spot.

D. Debbarma et al [8] compared the cooling performance of a channel with smooth walls and with two dimensions for the hemispherical protrusions. Their conclusions were that the protrusions added on the solid walls improve the heat transfer between the solid walls and fluid.

In order to evaluate the effect of the protrusions, the authors have simulated a uniform heat flux on the whole bottom surface of the cooling channel. Therefore, as said before, the position of the dimple is not important in overall cooling effect, because it will enhance thermal transfer in any position inside the cooler.

The cooling enhancement cause by the protrusions is already proven for a uniform heat flux. This paper wants to study the effect that a hemispherical protrusion has in cooling a smaller heat source compared to the cooling channel length. Using a heat source with dissipated power is similar to generating a hot spot next to the cooling channel. In this scenario, the position of the protrusion is influencing the heat source cooling.

B. Wu et al [9], J Turnow et al [10] and P. Xie et al [11] studied in different papers the fluid flow inside to cooling channels or cold plates. All of them used finite element analysis to investigate different geometries, optimize the existing design or observing multiple details that are hard to see during a physical test. Therefore finite element analysis seems to be a modern and useful technique to obtain the best results during the design phase.

\section{Finite element analysis of multiple cooling geometries}

\subsection{Cooling channel geometry}

To analyze the effect that different cold plates inside geometries have on cooling a heat source, finite element analysis were done starting from a base scenario. A simplified cooling channel was used, with the dimensions as in Fig 4.

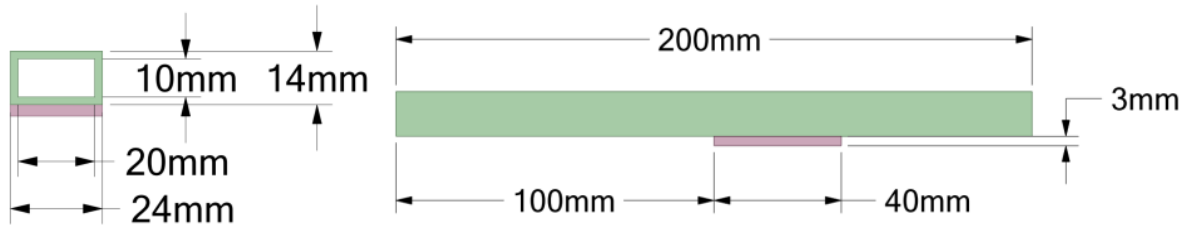

Fig. 4. Cooling channel dimensions

A heat source with the same width as the cooling channel of $24 \mathrm{~mm}, 3 \mathrm{~mm}$ thickness and $40 \mathrm{~mm}$ length was placed at $100 \mathrm{~mm}$ from the inlet, on the bottom exterior surface of the cooling channel. The dissipated power in the heat source is $120 \mathrm{~W}$.

To improve the cooling efficiency of the cooling channel, a hemispherical protrusion was place on the bottom surface. The dimensions of the geometry can be seen in Fig. 5 . 


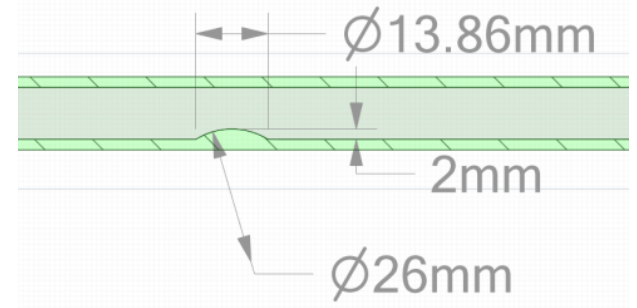

Fig. 5. Protrusion dimensions

In Table 1 the can be observed the total surface area in contact with the fluid for both simulated geometries. For the geometry with a protrusion, an increase of only $0.1 \%$ was made for the total surface in contact with the fluid. Therefore, the difference in cooling is not highly impacted by the increase in surface.

Table 1. Total surface comparison

\begin{tabular}{|l|c|c|}
\hline Version & Base design & Version 1 \\
\hline $\begin{array}{l}\text { Surface in contact with the fluid } \\
{\left[\mathrm{mm}^{2}\right]}\end{array}$ & 12000 & 12012.6 \\
\hline $\begin{array}{l}\text { Increase in surface area } \\
\text { compared to base design [\%] }\end{array}$ & - & 0.1 \\
\hline
\end{tabular}

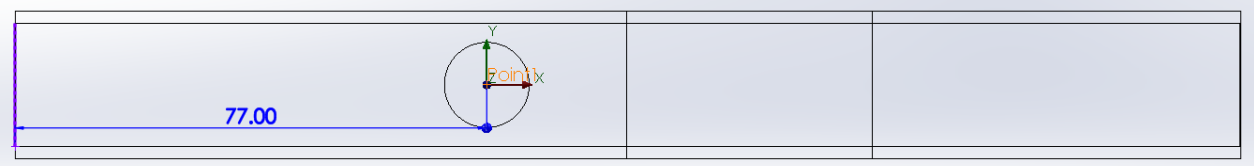

(a)

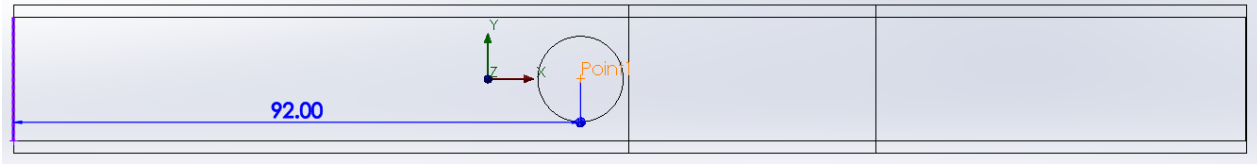

(b)

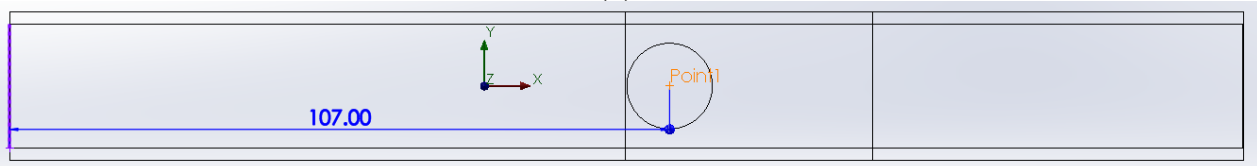

(c)

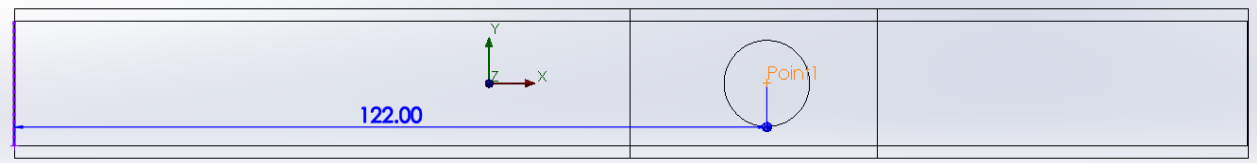

(d)

Fig. 6. a) Version A; b) Version B; c) Version C; d) Version D

In order to find the best-case scenario using this geometry, four different positions were used to place the geometry, based on the distance from the inlet area to the center of the sphere. The goal is to see how much a cooling channel can be optimized using only one protrusion and changing its position relative to the heat source and not implementing an array 
of additional geometries to highly increase the pressure drop. In Table 2 the distance from the inlet area to the center of the sphere can be observed.

Table 2. Distance from inlet to sphere center

\begin{tabular}{|l|c|c|c|c|}
\hline Version & Version A & Version B & Version C & Version D \\
\hline $\begin{array}{l}\text { Distance from } \\
\text { inlet area }\end{array}$ & $77 \mathrm{~mm}$ & $92 \mathrm{~mm}$ & $107 \mathrm{~mm}$ & $122 \mathrm{~mm}$ \\
\hline
\end{tabular}

In Fig. 6 the position of the sphere inside the cooling channel can be observed, based on the distance from the inlet surface area.

\subsection{Boundary conditions}

During the functioning of any car, environmental conditions change a lot. These changes are influenced by the location on Earth, by the season and even by day and night. When designing cooling solutions, the worst-case scenario should be taken into consideration. For these finite element simulations, the worst-case temperature values used in the automotive industry were used. Therefore, the ambient temperature of the cooling channel is $80^{\circ} \mathrm{C}$ and the cooling fluid inlet temperature is $65^{\circ} \mathrm{C}$.

In order to see only the influence of the cooling channel geometry, thermal radiation was not taken into consideration. All the thermal transfer was done due to thermal conduction and thermal convection.

Cold plate cooling solutions can be used for multiple types of automotive products, therefore the inlet flow rate can change due to the application or due to the cooling needed in a certain moment. To understand the cooling behavior and efficiency that the cold plate geometries presented above have, all the geometries were simulated with 4 different inlet flow rate values: $3 \mathrm{l} / \mathrm{min}, 5 \mathrm{l} / \mathrm{min}, 7 \mathrm{l} / \mathrm{min}$ and $9 \mathrm{l} / \mathrm{min}$. Cooling efficiency and pressure loss in influenced by the fluid velocity, hence flow rate has an influenced on those parameters. Therefore, a total number of 32 finite element simulation were solved.

\subsection{Mesh independent results}

In order to obtain consistent results for all the simulation, a mesh independent study was performed on the geometry with a protrusion implemented, considering that design to be more mesh demanding than the geometry with smooth walls. The software used for the simulations is Simcenter Flotherm XT 2019.3.

A surface inflation mesh with $0.5 \mathrm{~mm}$ element size was applied to the cooler inner surface to refine the mesh region in the boundary layer area. The surface mesh resulted on the protrusion and cooling channel surfaces can be seen in Fig. 7.

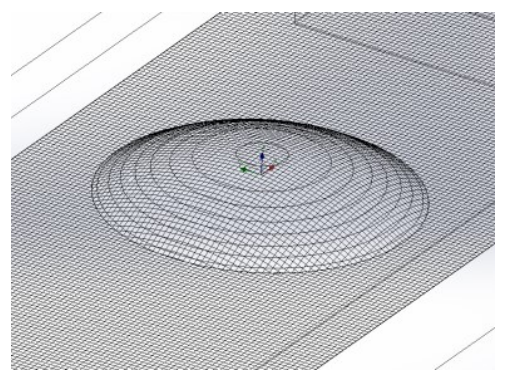

Fig. 7. Surface mesh on the protrusion 


\section{Simulation results}

\subsection{Base design simulations results}

The simulation results for the cooling channel without any geometry inside were considered the starting point in evaluating the efficiency of the other simulation scenarios. For an easier comparison of the simulation results, the average and maximum temperature of the heat source were evaluated, as well as the system pressure drop, to see also the negative effect that the geometries have on the cooling system.

The average temperature of the heat source when using a flow rate of $31 / \mathrm{min}$ is approximately $98,4^{\circ} \mathrm{C}$. Considering that the ambient temperature is $80^{\circ} \mathrm{C}$ and the dissipated power is $120 \mathrm{~W}$ we already see that fluid cooling is a very efficient cooling solution for high power components. In Table 3 can be observed the results for the average and maximum temperature of the heat source, as well the pressure loss values of the system.

Table 3. Heat source temperature and pressure loss for base design

\begin{tabular}{|l|c|c|c|c|}
\hline Fluid Flow [1/min] & 3 & 5 & 7 & 9 \\
\hline Heat source temp - Average $\left[{ }^{\circ} \mathrm{C}\right]$ & 98.4 & 93 & 89.2 & 88.7 \\
\hline Heat source temp - Max $\left[{ }^{\circ} \mathrm{C}\right]$ & 102.5 & 96.7 & 92.7 & 92.5 \\
\hline Pressure loss $[\mathrm{Pa}]$ & 14 & 28.3 & 50.1 & 76.4 \\
\hline
\end{tabular}

In Fig 8 can be observed the average temperature and maximum temperature evolution of the heat source, for all 4 flow rates. Between the first three flow rates we have almost a linear evolution. For the last flow rate, $9 \mathrm{l} / \mathrm{min}$, the temperatures are just slightly smaller than $7 \mathrm{l} / \mathrm{min}$. This values show us that for this design, that uses no inside geometry in the cooling channel, increasing the flow rate over a certain value will not bring significant improvement in cooling efficiency.

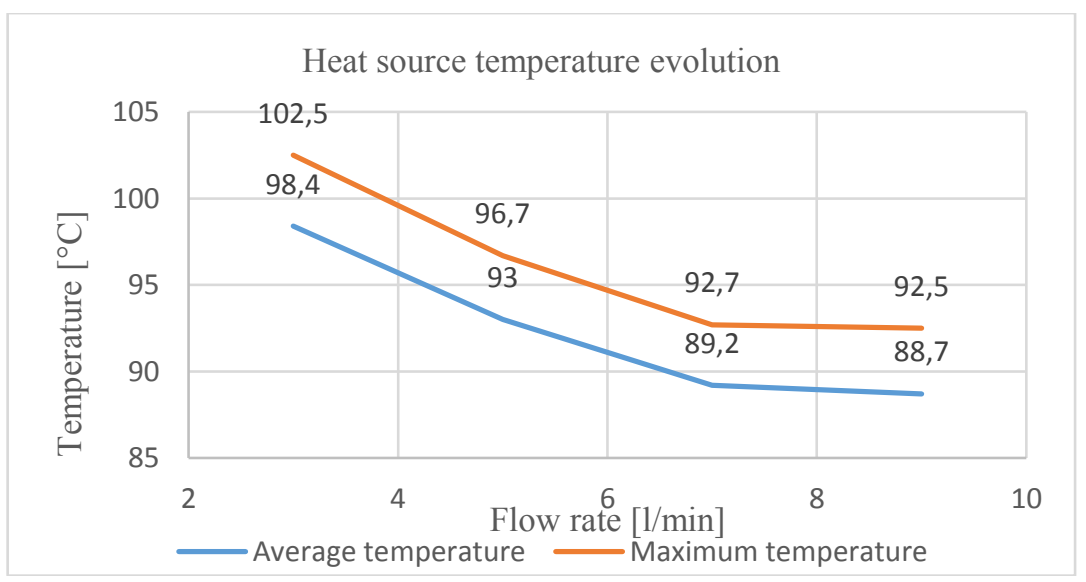

Fig. 8 Heat source temperature evolution for the base design

In Fig 9 the pressure loss values for each flow rate can be evaluated. Even if we saw that from 7 to $9 \mathrm{l} / \mathrm{min}$ the temperature reduction was not significant compared to the first flow rates, the pressure drop is not similar between the flow rates. 


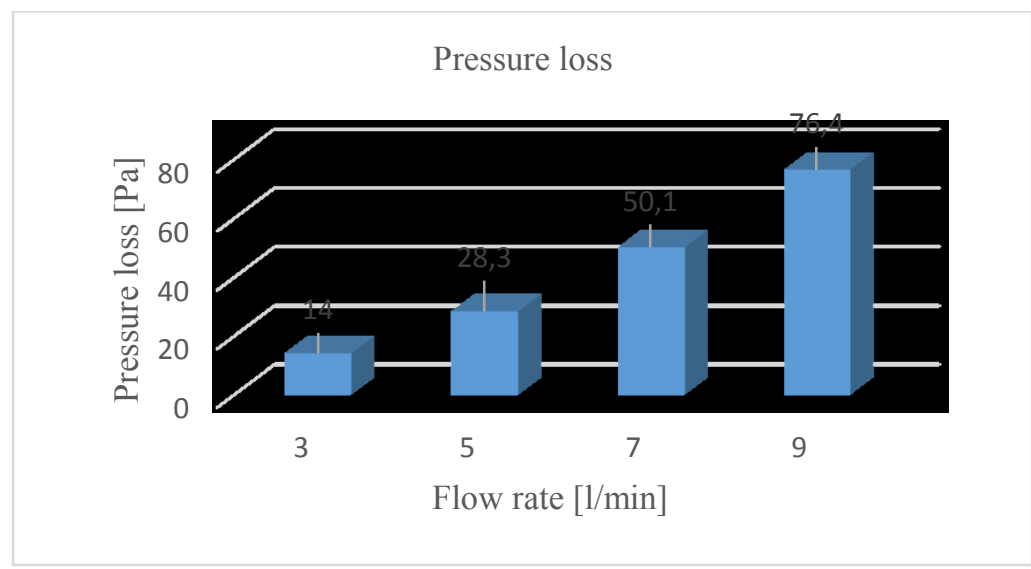

Fig. 9 Pressure loss value for the base design simulations

Comparing the pressure loss evolution with the heat source temperature evolution, we can say that the pressure loss evaluation cannot be the only evaluation factor in cooling systems efficiency.

\subsection{Geometry with protrusion simulations results}

Since the first improved version of the cooling channel is the addition of a small geometry, 4 different positions relative to the heat source were simulated, to observe if it has an impact on the heat source cooling. From the Table 4 we can see that the temperature of the heat source is influenced by the position of the added geometry.

Table 4. Temperature and pressure loss results for the simulations with protrusion

\begin{tabular}{|c|c|c|c|c|c|}
\hline Fluid Flow [1/min] & & 3 & 5 & 7 & 9 \\
\hline Heat source temp - Average $\left[{ }^{\circ} \mathrm{C}\right]$ & \multirow{3}{*}{$\begin{array}{c}\text { Version } \\
\text { A }\end{array}$} & 95.7 & 92.1 & 88.3 & 85.1 \\
\hline Heat source temp - Max $\left[{ }^{\circ} \mathrm{C}\right]$ & & 99.5 & 95.9 & 91.9 & 88.2 \\
\hline Pressure drop $[\mathrm{Pa}]$ & & 15.1 & 34 & 58 & 87.7 \\
\hline Heat source temp - Average $\left[{ }^{\circ} \mathrm{C}\right]$ & \multirow{3}{*}{$\begin{array}{c}\text { Version } \\
\text { B }\end{array}$} & 94.6 & 91.4 & 87.6 & 85.3 \\
\hline Heat source temp - Max $\left[{ }^{\circ} \mathrm{C}\right]$ & & 98.3 & 95.2 & 91.1 & 88.6 \\
\hline Pressure drop $[\mathrm{Pa}]$ & & 15.2 & 34.1 & 57.9 & 86.3 \\
\hline Heat source temp - Average $\left[{ }^{\circ} \mathrm{C}\right]$ & \multirow{3}{*}{$\begin{array}{c}\text { Version } \\
\mathrm{C}\end{array}$} & 93.1 & 89.4 & 85 & 82.9 \\
\hline Heat source temp - Max $\left[{ }^{\circ} \mathrm{C}\right]$ & & 96.4 & 93.5 & 88.6 & 86 \\
\hline Pressure drop $[\mathrm{Pa}]$ & & 15.3 & 34.1 & 58.2 & 87.6 \\
\hline Heat source temp - Average $\left[{ }^{\circ} \mathrm{C}\right]$ & \multirow{3}{*}{$\begin{array}{c}\text { Version } \\
\text { D }\end{array}$} & 93.7 & 88.8 & 85.1 & 83.7 \\
\hline Heat source temp - Max $\left[{ }^{\circ} \mathrm{C}\right]$ & & 95.9 & 90.7 & 87.7 & 86.9 \\
\hline Pressure drop $[\mathrm{Pa}]$ & & 15.4 & 33.6 & 57.8 & 88.5 \\
\hline
\end{tabular}

In Fig. 10 the evolution of the heat source average temperature can be observed. For all 4 scenarios, the temperature is decreasing with the rise of the flow rate. For the smallest simulated flow rate, of $3 \mathrm{l} / \mathrm{min}$, and the highest simulated flow rate, of $9 \mathrm{l} / \mathrm{min}$, the difference between the maximum and minimum average temperature is smaller compared to the 
simulations done with $5 \mathrm{l} / \mathrm{min}$ and $7 \mathrm{l} / \mathrm{min}$. The conclusion is that this kind of geometry should be optimized for smaller variation is flow rate, not for all the possible scenarios. We can also conclude that the best position to place the additional geometry is as close as possible to the heat source, inside the cooling channel. This shape placed inside the cooling channel has the effect of reducing the fluid boundary layer, which means an improved cooling on the surface next to the heat source.

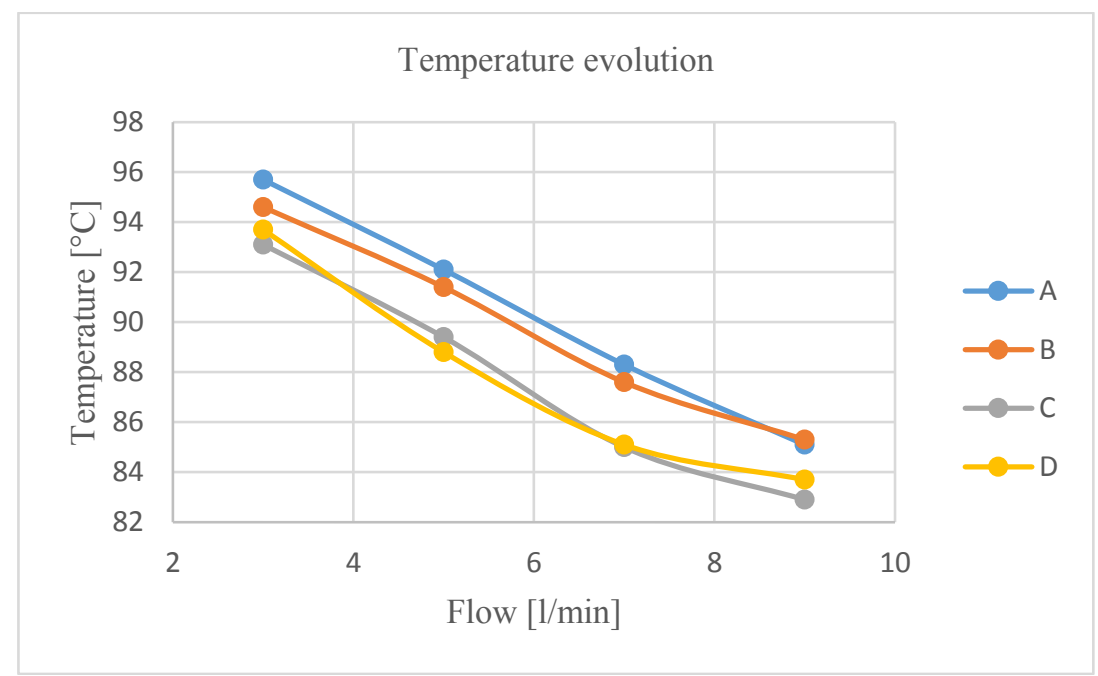

Fig. 10. Average temperature evolution of the heat source

The pressure loss for all the scenarios of the geometry with protrusion are very close to each other, as can be seen in Fig. 11. Very small differences are caused by the relative position of the additional geometry regarding the inlet and outlet areas, but as expected, the pressure loss values are similar to each other.

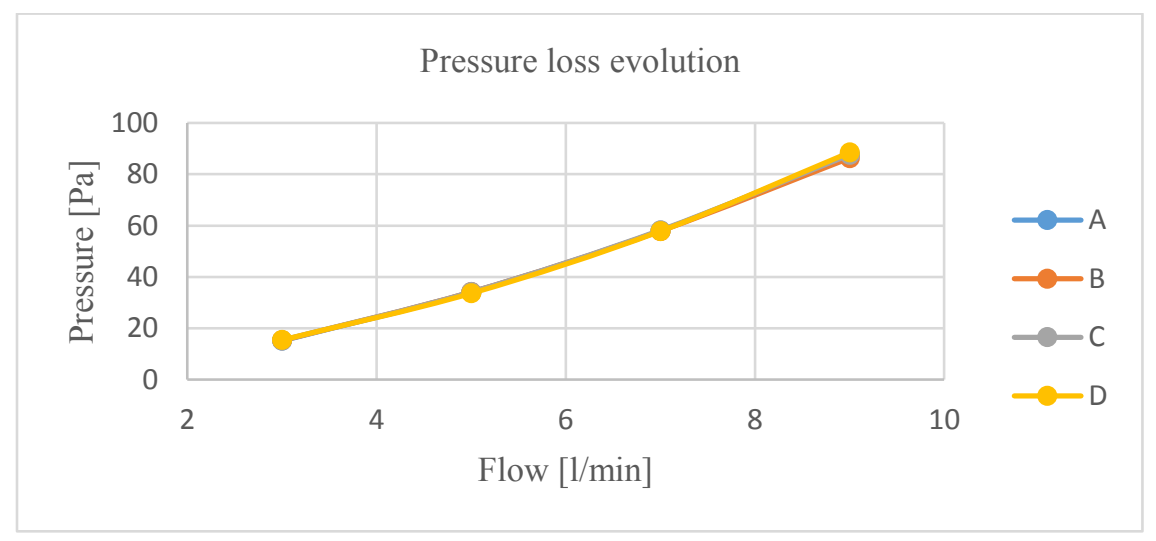

Fig. 11. Pressure loss evolution of the simulations with protrusion

\subsection{Results comparison}

In Fig. 12 the average temperature evolution of the heat source can be seen for all the simulated scenarios. For all the fluid flow rates, we can see a decrease in the temperature values from the base design. Considering that the ambient temperature is $80^{\circ} \mathrm{C}$ and the fluid 
inlet temperature is $65^{\circ} \mathrm{C}$, reducing $5.8^{\circ} \mathrm{C}$ in the average temperature for Version $\mathrm{C}$ at $9 \mathrm{l} / \mathrm{min}$ fluid flow is a significant reduction obtained with only one protrusion added.

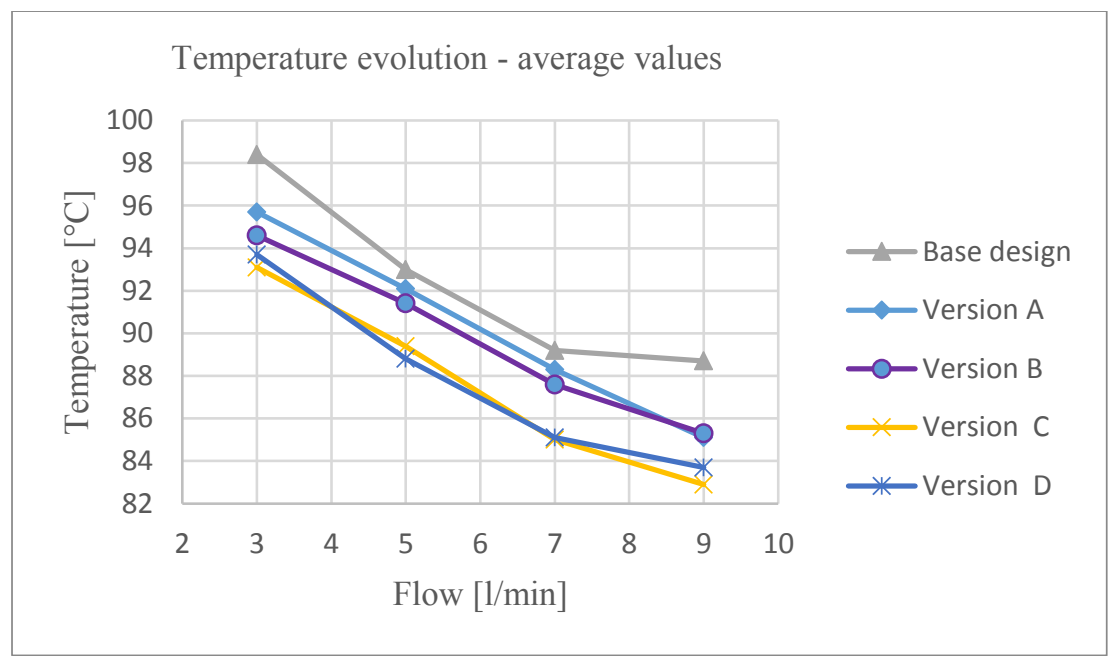

Fig. 12. Heat source average temperature evolution for all the simulation scenarios

Even if the increase in surface area is not significant from the base design to the geometry with protrusion added, the pressure loss growth is higher. The smaller increase in pressure loss can be observed at $3 \mathrm{l} / \mathrm{min}$ fluid flow rate, with an increase of $7.9 \%$. The highest increase in pressure drop is at $5 \mathrm{1} / \mathrm{min}$ flow rate, where we saw an increase of $20.1 \%$ in the pressure loss value. All the values for the pressure loss and increase compared with the base design can be seen in Table 5 .

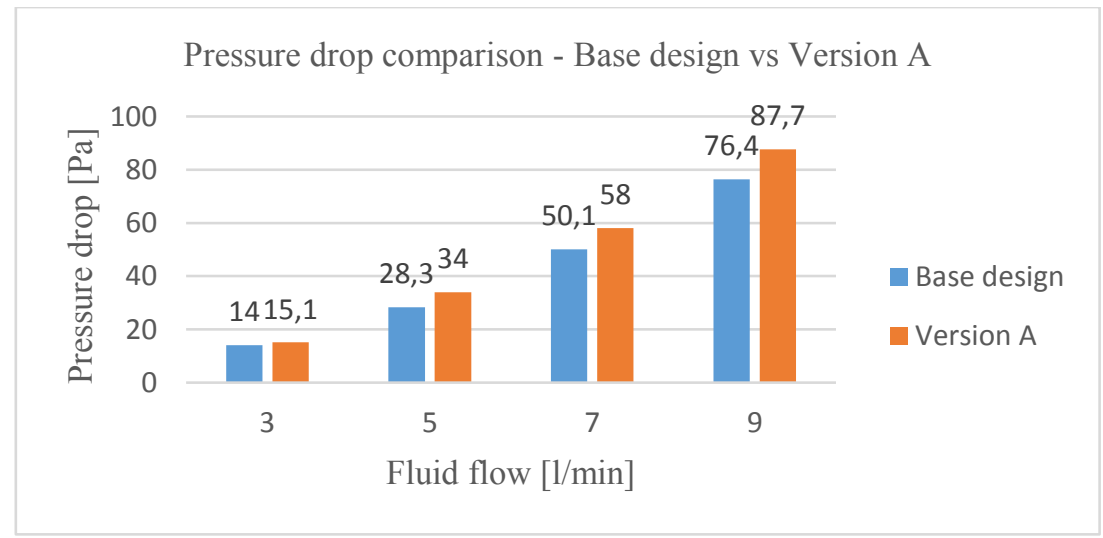

Fig. 13. Pressure loss comparison between Base Design and Version A

Table 5. Pressure loss values and increase in \%

\begin{tabular}{|l|c|c|c|c|}
\hline Fluid Flow [1/min] & 3 & 5 & 7 & 9 \\
\hline Pressure drop [Pa] - Base design & 14 & 28.3 & 50.1 & 76.4 \\
\hline Pressure drop [Pa] - Version A & 15.1 & 34 & 58 & 87.7 \\
\hline Increase in pressure drop [\%] & 7.9 & 20.1 & 15.8 & 14.8 \\
\hline
\end{tabular}


In Fig. 14 we can see the fluid speed inside the cooling channel for the base scenario simulation at $3 \mathrm{l} / \mathrm{min}$ fluid flow. As expected, the fluid velocity next to the cooling channel walls is reduced compared to the maximum velocity, therefore, the cooling capabilities are reduced.

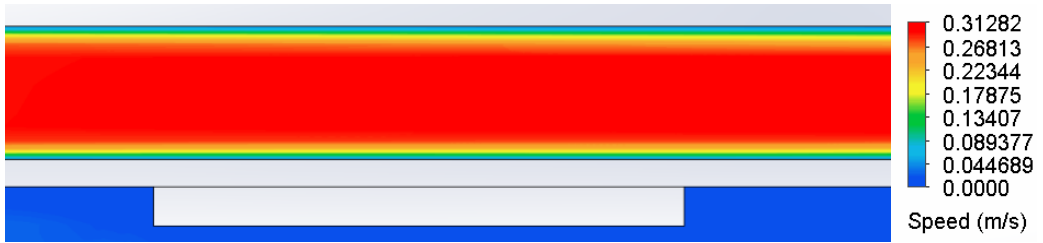

Fig. 14. Fluid speed inside the cooling channel for base scenario

In Fig. 15 both the benefits and downside of the protrusion can be seen. The velocity of the fluid next to the bottom wall, before the protrusion is small and the boundary layer is visible. When the fluid hits the protrusion, the boundary layer decreases and the fluid velocity increases. Here is where the highest heat flux can be seen. Right after the protrusion, the fluid velocity is very small, because of the turbulent movement of the fluid. Also the boundary layer thickness is higher than before. The downside of this positioning of the protrusion is that the boundary layer thickness is very high right on top of the heat source.

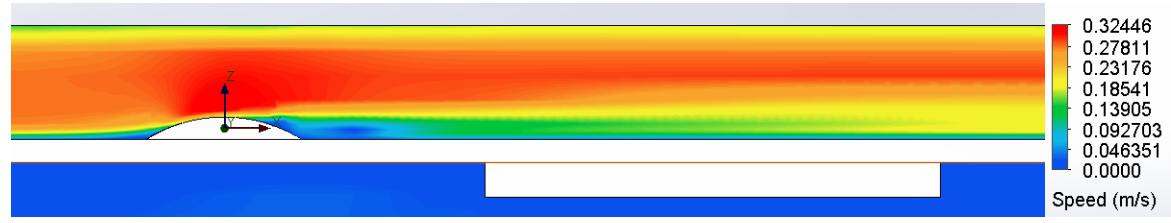

Fig. 15. Fluid velocity for Version A at $31 /$ min fluid flow rate

In Fig 16 the same fluid behavior can be seen, with similar velocity. In this scenario, the temperature of the heat source is smaller, not because the fluid is moving in a different manner, but because the protrusion is closer to the heat source, therefore, more heat from the source can reach the protrusion. The temperature difference between the fluid and protrusion is higher than in design $\mathrm{A}$, therefore the heat transfer is improved even more.

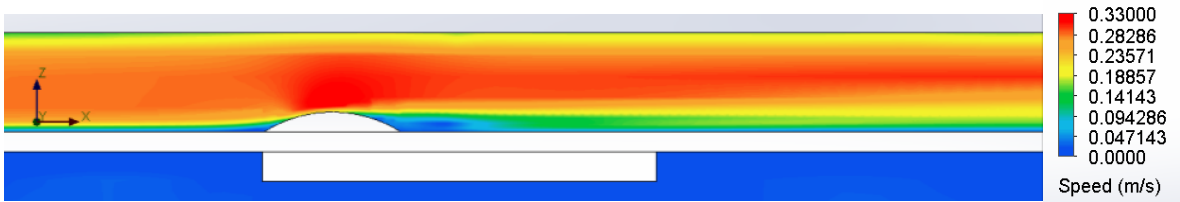

Fig. 16. Fluid velocity for Version $C$ at $31 /$ min fluid flow rate

In Fig 17 the fluid temperature can be seen in the same section plane as the velocity. This shows us, that right after the protrusion, where we have a small fluid velocity, also the temperature of the fluid is increased. The turbulent behavior from the fluid flow right after the protrusion is not improving the cooling of the cooling channel wall. 


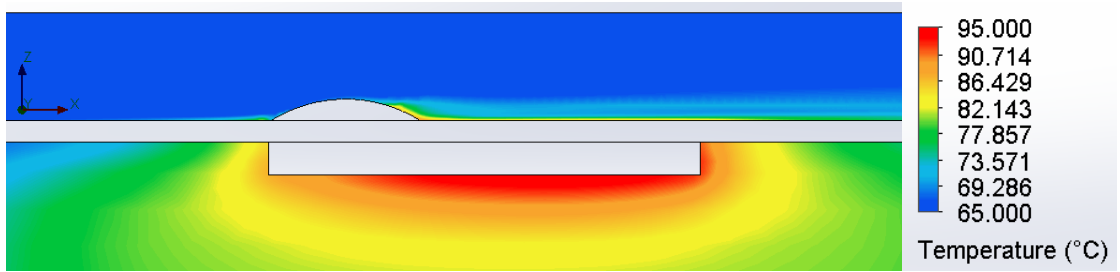

Fig. 17. Fluid temperature for Version $\mathrm{C}$ at $31 / \mathrm{min}$ fluid flow rate

Saw from the top, as in Fig. 18, the temperature plot shown us that the front of the protrusion is cooled better than the back side, as we already been able to see from the side section view.

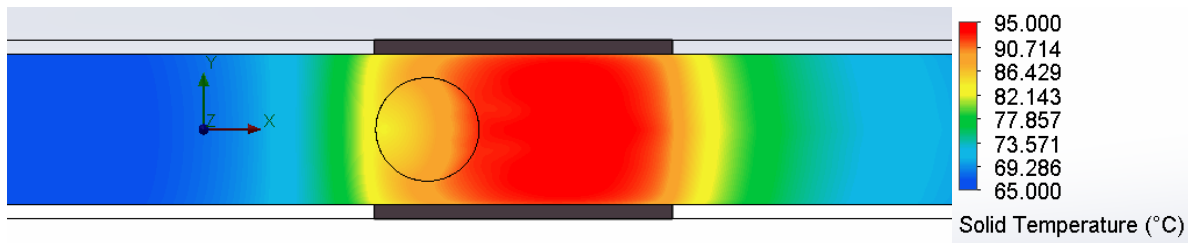

Fig. 18. Temperature plot on bottom surface for Version $\mathrm{C}$ at $3 \mathrm{l} / \mathrm{min}$ flow rate

In Fig. 19 and Fig 20 we can see the particles plot around the protrusion. We already saw that the fluid velocity is decreasing right after the fluid passes the protrusion. This is because the fluid that moves around the protrusion in reaching the area with a lower pressure, behind the protrusion, creating turbulences.

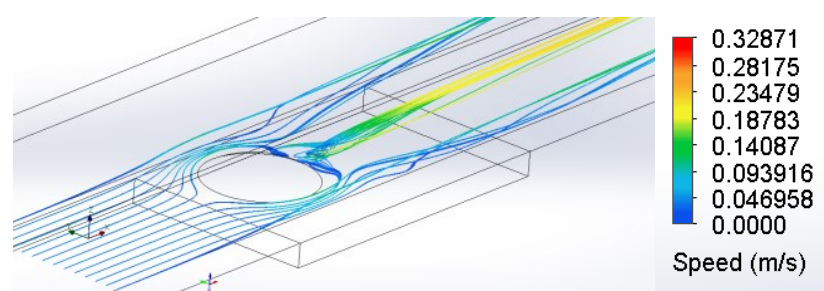

Fig. 19. Particle plot velocity for Version $C$ at $31 / \mathrm{min}$

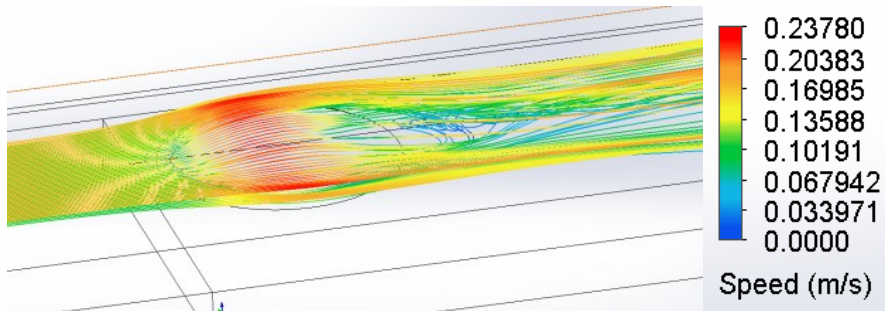

Fig. 20. Particle plot velocity for Version $\mathrm{C}$ at $31 / \mathrm{min}$

In the side section view, the temperature particle plot, as in Fig 21 shows us that the fluid temperature that is reaching the protrusion is colder than the fluid next to the cooling channel walls. In Fig. 22 we can see again that the velocity of the fluid that is reaching the protrusion is higher than the one from the boundary layer. The increase in velocity is mixing the hot and cold fluid, improving the cooling of the protrusion surface. 


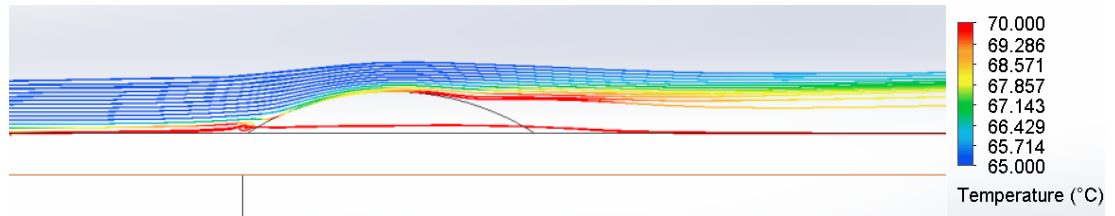

Fig. 21. Section view of temperature particle plot at $31 / \mathrm{min}$

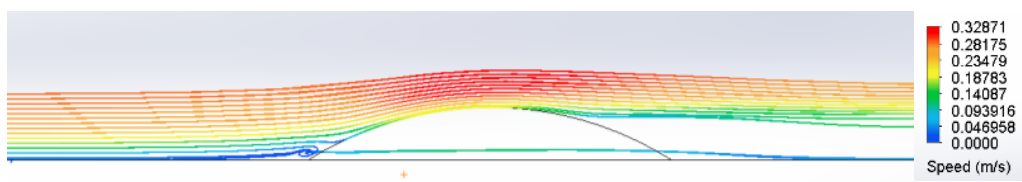

Fig. 22. Section view of particle plot velocity at $31 / \mathrm{min}$

\section{Conclusions}

We already knew from the existing research that an array of protrusions generate an improvement in cooling, when placed on a surface with a continuous heat flux, but also an increase pressure loss inside the system. This paper studied the effect that only one protrusion has on a heat source that is creating a hot spot on the bottom surface of the cooling channel. Four different positions were considered for the protrusion and the results were compared with the base design, that had no protrusion inside the cooling channel.

The results has shown that the added protrusion on the bottom surface of the cooling channel will improve the cooling of the heat source. The heat transfer from the solid to fluid is improved, regardless of the protrusion position.

The results also shown that the temperature values are different based on the position of the protrusion inside the cooling channel. Therefore, in order to get the best cooling results with a small increase in pressure loss, single protrusions with an optimized position may be used to improve the cooling over hot spots.

In this phase of the research, an optimized position of the protrusion is considered as close as possible to the hotspot area or close to the heat source. If the area with a high dissipated power is higher compared to a protrusion area, an array of protrusions may be more efficient. Because any case of fluid cooling has its particularities and requirements, these conclusions can be used as a starting point in designing and simulating an efficient cold plate.

\section{References}

1. R. K. Shan, D. P. Sekulic, Fundamentals of Heat Exchanger Design (John Wiley \& Sons, New Jersey, 2003)

2. D. Marghitu, Mechanical Engineer's Handbook (Academic Press, California, 2001)

3. J. Lee, K. S. Lee, International Journal of Heat and Mass Transfer, 64, 444-451 (2013)

4. R.K. Rajput, Engineering Thermodynamics Third Edition (Laxmi Publications Ltd., New Delhi, 2007)

5. M. Faizan, S.Z. Shuja, B.S. Yilbas, M. Khan, H. Al-Qahtani, International Journal of Thermal Sciences, 162, 106793 (2021)

6. Y. Xie, H. Qu, D. Zhang, International Journal of Heat and Mass Transfer, 84, 486 $496(2015)$

7. J. Liu, Y. Song, G. Xie, B. Sunden, Energy, 79, 1-19 (2015) 
8. D. Debbarma, K. M. Pandey, A. Paul, Materials Today: Proceedings, (2021)

9. B. Wu , X. Yang, Z. Liu , Z. Feng, International Communications in Heat and Mass Transfer, 121, 105072 (2021)

10. J. Turnow , N. Kornev, V. Zhdanov, E. Hassel, International Journal of Heat and Fluid Flow, 35, 168-175 (2012)

11. P. Xie, X. Zhang, International Communications in Heat and Mass Transfer, 120, $105030(2021)$ 